\title{
Atomistic measures of mechanical deformation and thermal transport processes
}

\author{
Sidney Yip*, Ju Li, Wei Cai, Jinpeng Chang, Dongyi Liao \\ Department of Nuclear Engineering, Room 24-208, Massachusetts Institute of Technology, Cambridge, MA 02139-4307, USA
}

\begin{abstract}
We discuss several atomistic simulation results on the study of ideal strength, dislocation mobility and thermal resistance in crystalline solids as contributions to the implementation of multiscale materials modeling. We also emphasize the need to link the atomistic approach to mesoscale simulation techniques in order to address microstructural complexities at larger length and time scales.
\end{abstract}

Keywords: Please provide 3-5 keywords

Currently much recognition is being given to an area of computational materials research that has come to be known as multiscale materials modeling [1-4]. This is an approach in which theoretical concepts and simulation techniques appropriate to different length (and time) scales are combined with selected experiments to enable a more robust analysis and prediction of certain materials properties and behavior. In this paper, which is primarily tutorial in nature, we examine the contributions that atomistic simulations can make toward realizing the potential of multiscale materials modeling using examples of our current studies of mechanical deformation and thermal conduction in crystalline solids.

We begin by noting that among the several powerful drivers of the growing interest in developing computational models of materials performance are the advent of high performance computing, the holistic view of molecular engineering in the development of advanced materials, the emergence of nanoscience and technology, and a number of grand challenge projects such as the ASCI-Dislocation Dynamics initiative at the Lawrence Livermore National Laboratory [5] and the DOE Computational Materials Science Network thrust on grain boundary-dislocation interactions [6]. As the field is evolving, materials modeling has emerged to be a highly multidisciplinary enterprise, emphasizing the value of team research and the exploitation

\footnotetext{
* Corresponding author: Tel.: +1 (617) 253-3809; Fax: +1 (617) 258-8863; E-mail: syip@mit.edu
}

of complementary skills and expertise among the collaborators.

That materials modeling is inherently a multiscale problem is clear to anyone who has considered a certain materials behavior, say mechanical deformation of a solid, in terms of typically four distinct levels of microstructural complexity, the electronic-structure, the atomistic, the mesoscale, and the continuum. At each level there exist corresponding methods of simulation, first-principles quantum mechanical calculations, classical molecular dynamics and Monte Carlo simulations, finite-element methods, and continuum mechanics (see Fig. 1). Many, though not all, practical applications involve continuum or finite-element calculations that require material-specific parameters or properties obtainable only by atomistic or ab initio cal-

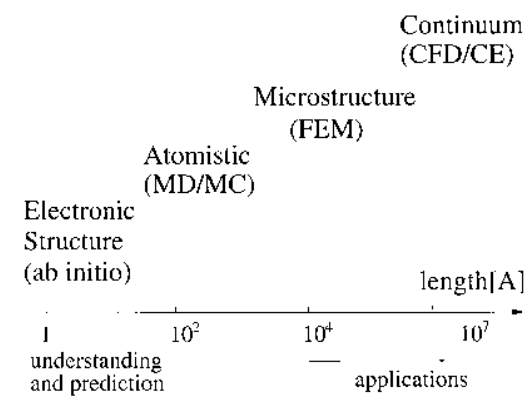

Fig. 1. The four characteristic length scales in multiscale materials modeling. 
culations, or derived from experiments. Thus the effective linking of different methods in multiscale modeling is recognized to be a particular challenge, especially the connection between atomistic and the mesoscale levels [7,8].

In the present discussion our interest lies at the atomistic scale where the material system is treated as an assembly of atoms that interact with each other according to interatomic forces derived from prescribed model potential functions $[9,10]$. Perhaps the most valuable aspect of this approach is that one does not a priori specify the mechanism by which the system evolves, or in other words atomistic simulations are capable of revealing the detailed structure and dynamics of interatomic interactions on length scales ranging from clusters of a few atoms to larger units up to the size of the simulation cell. Present practical limitations on system size and simulation time are of the order of a few million atoms and a microsecond, although simulations using much larger number of atoms or running much longer have been made under very special circumstances. It is also well recognized that atomistic simulations depend on the reality of interatomic potential used for their accuracy. As progress is made in developing potential functions which are physically realistic and computationally still tractable, the limitation associated with inadequate potential models becomes less severe, leading to a larger class of materials systems that can be studied by atomistic simulations.

To illustrate the foregoing remarks we consider the atomistic approach to strength determination under various modes of deformation. This is a classic problem of fundamental as well as technological interest. One can expect that the maximum resistance to deformation of an assembly of interacting atoms must bear close relation to the cohesive energy of the system, and that this property ought to be directly measurable in a simulation. We therefore imagine applying an incremental load to our simulation cell through a sequence of prescribed strains and then observing the resulting change in the stress in the sample. Using a single crystal of initially defect-free $\beta$-SiC and applying hydrostatic tension at room temperature, one obtains a stress-strain response shown in Fig. 2 [11]. In this simulation a particular interatomic potential model which treats the $\mathrm{SiC}$ as a purely covalent material [12] is used, the specific details of the interaction are of no interest in the present illustrative discussion. While the initial portion of the stress-strain curve is not plotted, it can be safely taken to show the familiar linear elastic response which connects onto the indicated nonlinear but still elastic region. The abrupt change at a critical strain just beyond $15 \%$ signifies an instability. Inspecting the detailed atomic configurations available in the simulation data one sees that a small crack has nucleated in the severely strained lattice, and subsequent deformation upon further straining is localized around the crack opening. Repeating the simulation with two other prepared initial structures, an amorphous and a nanocrystalline sample, one finds the results are qual-

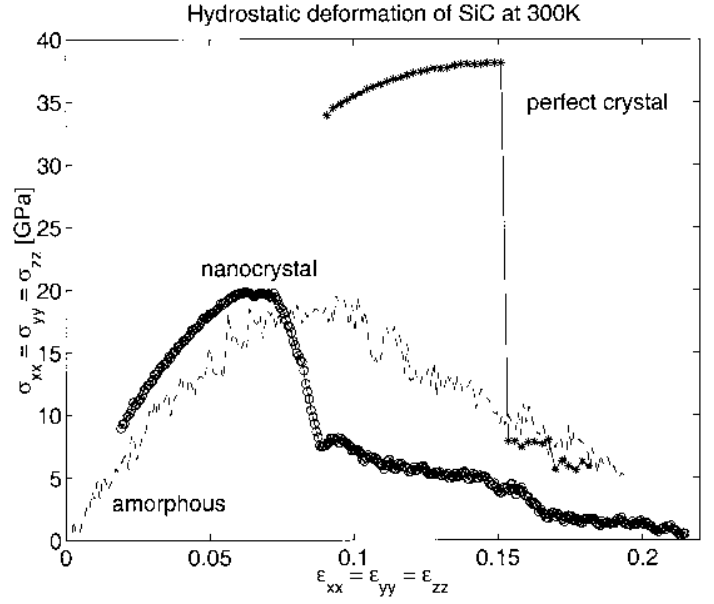

Fig. 2. Response of $\mathrm{SiC}$ in single crystal, amorphous, and nanocrystalline form to constant hydrostatic strain deformation.

itatively rather similar with regard to their deviations from the single crystal behavior.

Fig. 2 shows that our model of $\beta$-SiC has a theoretical strength in hydrostatic tension of 38,22 , and 20 GPA for the single crystal, amorphous, and nanocrystalline structures respectively. It is to be expected that the single crystal strength sets the upper limit, since any defect in the lattice will act as a stress concentration and thereby lower the system resistance. The behavior of the amorphous and the nanocrystalline responses is rather revealing. The particular nanocrystalline sample used in the simulation is a very small system, consisting of only 4 grains in a periodic cell, each with a diameter of about $4 \mathrm{~nm}$. The similarity observed here suggests a possible close connection between the nanocrystal in the limit of very small grains and the amorphous state.

The homogeneous deformation of a uniform lattice without defects is a problem for which elastic stability criteria have been derived [13-15]. For a cubic lattice under an applied hydrostatic pressure $P(P<0$ for tension), the stability conditions require the bulk and two shear moduli to be positive,

$$
\begin{aligned}
& B=\left(C_{11}+2 C_{12}+P\right) / 3>0, \\
& G^{\prime}=\left(C_{11}-C_{12}-2 P\right) / 2>0, \\
& G=C_{44}-P>0,
\end{aligned}
$$

where $C_{i j}$ are the elastic constants at the current state. A variety of direct simulation studies [16] have shown that these criteria do accurately describe the critical value of $P$ at which the homogeneous lattice becomes unstable. One may therefore regard such critical values as the ideal or theoretical strength for the particular mode of loading. The extension of stability considerations to the more general behavior of lattice vibrations has been made by the analysis 
of phonon dispersion curves for deformed lattices, thus allowing for the possibility of onset of soft modes other than longitudinal or transverse acoustic vibrations at $\mathbf{k}=0$ [11].

The mechanistic understanding of how crystalline materials deform under an imposed load requires knowledge of mobility of extended defects such as dislocations and grain boundaries. In the context of single crystal plasticity, molecular dynamics and the mesoscale simulation technique of 'dislocation dynamics' are now being applied to elucidate the core processes of interatomic interactions and the long-range elastic interactions respectively [17]. The issue of connecting the two levels of simulation is also beginning to receive attention [7]. In crystals with high lattice resistance to dislocation motion, dislocation mobility is commonly described in terms of the double-kink mechanism. We have formulated a kinetic Monte Carlo treatment of kink nucleation, migration, and annihilation processes along with full elastic interactions and obtained results that can be compared directly with measurements on screw dislocations in $\mathrm{Si}$ [8]. The formulation is designed to produce the overall dislocation movement as the cumulative effect of a large number of individual events, requiring for input only the kink formation and migration energies that are determined by atomistic calculations. As such, it is an example of multiscale modeling linking the atomistic and the meso scales. Under favorable conditions, it is feasible to observe directly the dislocation motion through molecular dynamics simulations. Data on dislocation velocity of edge dislocations in bcc Mo reveal evidence of natural kink

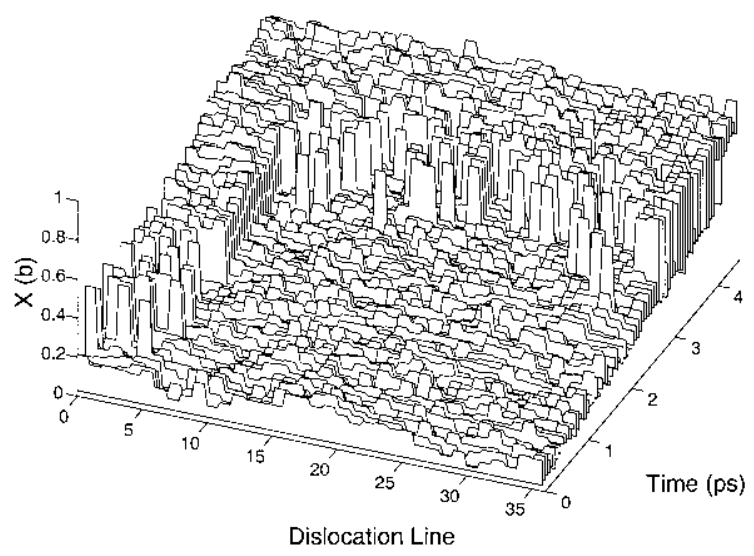

Fig. 3. Motion of a dislocation line in a bcc metal (molybdenum) consisting of intermittent nucleation of short kink segments as revealed by molecular dynamics simulation.

formation (see Fig. 3), and from the observed temperature dependence the characteristic damping behavior of phonon drag [18].

Since atomistic simulation is equally well suited to probing thermal phenomena, we discuss briefly a study of the lattice thermal conductivity of $\beta$-SiC employing the same potential that was used to produce Fig. 2. From linear response theory in statistical mechanics, molecular expressions have been derived for various time-dependent current correlations functions whose time integral give the transport coefficients such as the thermal conductivity, shear

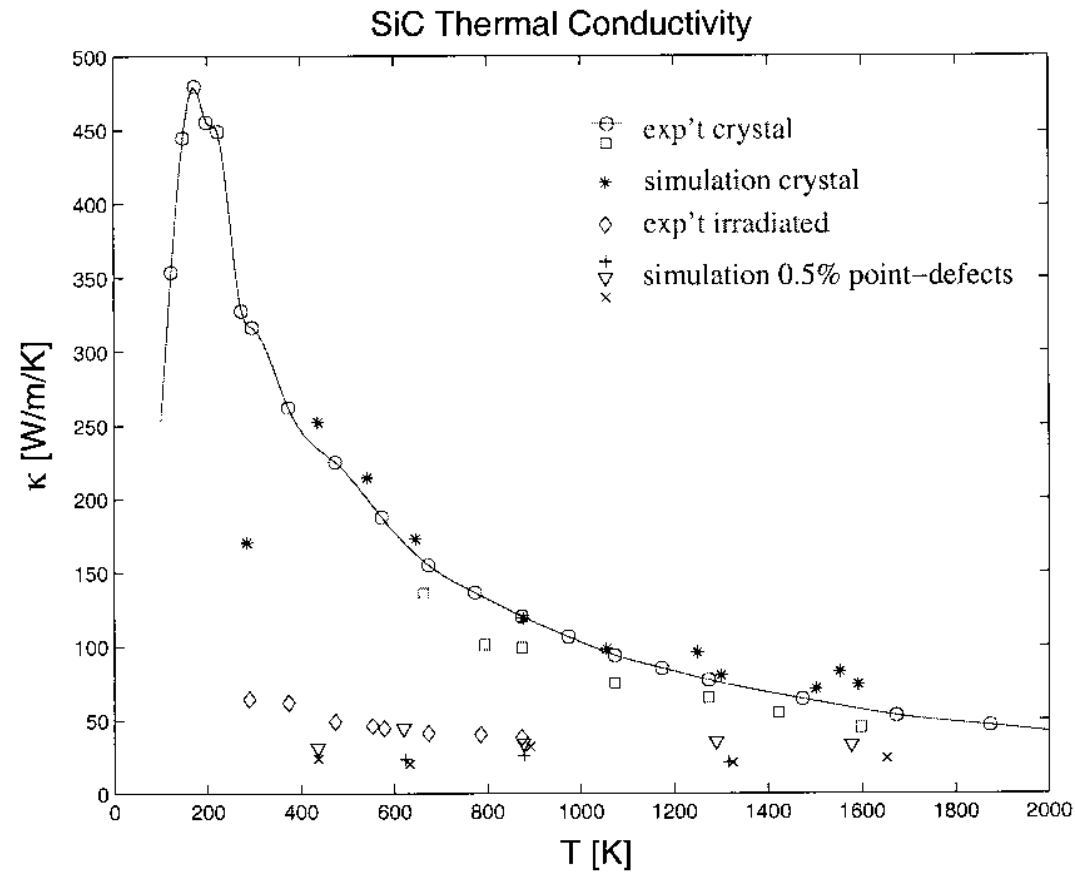

Fig. 4. Thermal conductivity of $\mathrm{SiC}$ without and with point defects at a concentration of $0.5 \%$. MD simulation and experiments. 
viscosity, and the diffusion coefficient. For fluids this approach is now quite familiar where the correlation functions are obtained by molecular dynamics simulation. We have shown that the formalism is also applicable to solids. In the case of single crystal of the simulation results above the Debye temperature are in quite satisfactory agreement with experiment, showing approximately an inverse temperature variation that is characteristic of phonon scattering (see Fig. 4) [19]. When point defects were introduced into the sample, the conductivity was found to decrease markedly along with the loss of temperature dependence, a behavior which is in accord with measurements on specimen that have been damaged by neutron and ion irradiation.

We have described a few results in mechanical deformation and thermal transport to illustrate how atomistic simulations can contribute to the fundamental understanding of materials behavior. Besides the problems of developing robust interatomic potentials for materials of greater chemical complexity and techniques for probing microstructural processes at the atomistic level, there are challenges in the implementation of multiscale modeling. A particularly central issue is the effective coupling of an atomistic region to its surrounding which is described on a more coarse scale $[20,21]$.

\section{Acknowledgements}

This work was supported by the Air Force Office of Scientific Research (F49620-00-10082), National Science Foundation (DMR-9980015), and Lawrence Livermore National Laboratory (B338297). URL: mmm.mit.edu.

\section{References}

[1] Campbell GH et al. Multi-scale modeling of polycrystal plasticity: a workshop report. Mater Sci Eng A 1998;251:122.

[2] Yip S (Ed). Proceedings of Workshop on Modeling of Industrial Materials: Connecting Atomistic and Continuum Scales. J Computer-Aided Mater Des 1996;3.

[3] Kaxiras E, Yip S. Mutliscale aspects of physics of materials: mechanical response, processing and new materials. Curr Opin Solid State Mater Sci 1998;3:523-525.

[4] Bulatov VV, Diaz de la Rubia, Phillips R, Ghoniem N (Eds). Multiscale Modeling of Materials. MRS Symposium Proceedings Series, vol 538, 1999.
[5] Mailhiot C. The DOE Accelerated Strategic Computing Initiative: Challenges and opportunities for predictive materials simulation capabilities. J Computer-Aided Mater Des 1998;5:95-107.

[6] Computational Materials Science Network, http://www. msd.anl.gov/im/cmsn/cmsn.html.

[7] Bulatov V, Abraham FE, Kubin L, Devincre B, Yip S. Connecting atomistic and mesoscopic simulations of crystal plasticity. Nature 1998;391:669-672.

[8] Cai W, Bulatov VV, Justo JF, Argon AS, Yip S. Intrinsic mobility of a dissociated dislocation in silicon. Phys Rev Lett 2000;84:3346-3349.

[9] Yip S. Atomistic modeling of liquids. The Encyclopedia of Advanced Materials. Pergamon, pp. 161-168, 1994.

[10] Yip S. Atomistic simulations in materials science. In: Ciccotti G, Hoover WG (Eds), Molecular-Dynamics Simulation of Statistical Mechanical Systems. Amsterdam: NorthHolland, pp. 523-556, 1986.

[11] Li J. Modeling Micorstructural Effects on Deformation Resistance and Thermal Conductivity. Ph.D. Thesis, Massachusetts Institute of Technology, Department of Nuclear Engineering, 2000.

[12] Tersoff J. Modeling solid-state chemistry: Interatomic potentials for multicomponent systems. Phys Rev B 1989;39:5566-5568.

[13] Wang J, Li J, Yip S, Phillpot S, Wolf D. Mechanical Instabilities of Homogeneous Crystals. Phys Rev B 1995;52:12627-12635.

[14] Zhou Z, Joos B. Stability criteria for homogeneously stressed materials and the calculation of elastic constants. Phys Rev B 1996;54:3841-3850.

[15] Morries JW, Krenn CR. The internal stability of an elastic solid. Philos Mag A, in press.

[16] Yip S, Li J, Tang, M, Wang J. Mechanistic aspects and atomic-level consequences of elastic instabilities in homogeneous crystals. Mater Sci Eng A, in press.

[17] Bulatov VV, Kubin L. Dislocation modelling at atomistic and mesoscopic scales. Curr Opin Solid State Mater Sci 1998;6:558-561.

[18] Chang J, Bulatov VV, Yip S. Molecular dynamics study of edge dislocation motion in a bcc metal. J Computer-Aided Mater Des 1999;6:165-173.

[19] Li J, Porter LJ, Yip S. Atomistic modeling of finitetemperature properties of crystalline $\beta$-SiC: II. Thermal conductivity and effects of point defects. J Nucl Mater 1998;255:139-152.

[20] Phillips R. Multiscale modeling in the mechanics of materials. Curr Opin Solid State Mater Sci 1998;6:526-532.

[21] Cai W, de Koning, Bulatov VV, Yip S. Minimizing boundary reflections in coupled-domain simulation. Phys Rev Lett 2000;85:3213-3216. 